\title{
The broad-band X-ray spectrum of the Seyfert 1 galaxy, MCG+8-11-11
}

\author{
S. Bianchi ${ }^{1}$, I. De Angelis ${ }^{1}$, G. Matt ${ }^{1}$, V. La Parola ${ }^{2}$, A. De Rosa ${ }^{3}$, P. Grandi ${ }^{4}$, E. Jiménez Bailón ${ }^{5}$, and E. Piconcelli ${ }^{6}$ \\ 1 Dipartimento di Fisica, Università degli Studi Roma Tre, via della Vasca Navale 84, 00146 Roma, Italy \\ e-mail: bianchi@fis.uniroma3.it \\ 2 INAF, Istituto di Astrofisica Spaziale e Fisica Cosmica, via U. La Malfa 153, 90146 Palermo, Italy \\ 3 INAF/IASF-Roma, via del Fosso del Cavaliere, 00133 Roma, Italy \\ 4 INAF-IASF Bologna, via Gobetti 101, 40129 Bologna, Italy \\ 5 Instituto de Astronomía, Universidad Nacional Autónoma de México, Apartado Postal 70-264, 04510 Mexico DF, Mexico \\ ${ }^{6}$ Osservatorio Astronomico di Roma (INAF), via Frascati 33, 00040 Monte Porzio Catone, Italy
}

Received 14 June 2010 / Accepted 9 July 2010

\section{ABSTRACT}

\begin{abstract}
Aims. Evidence is mounting that the main ingredients of the unification models of active galactic nuclei may behave differently from expectations, and be intimately related to fundamental physical parameters. The availability of high signal-to-noise broad-band X-ray spectra provides us with the opportunity to study in detail all the contributions from the materials invoked in these models, and infer their general properties, including whether their presence or absence is related to other quantities.

Methods. We present a long (100 ks) Suzaku observation of one of the X-ray brightest AGN, MCG+8-11-11. These data are complemented with the 54-month Swift BAT spectrum, allowing us to perform a broad-band fit to the $0.6-150 \mathrm{keV}$ range.

Results. The fits performed in the $0.6-10 \mathrm{keV}$ band provide results consistent with those of a previous XMM-Newton observation, i.e. a lack of a soft excess, warm absorption along the line of sight, a large Compton reflection component $(R \simeq 1)$, and an absence of a relativistic component of the neutral iron $\mathrm{K} \alpha$ emission line. However, when the PIN and Swift BAT data are included, the reflection amount drops significantly $(R \simeq 0.2-0.3)$, and a relativistic iron line is required, the latter being confirmed by a phenomenological analysis in a restricted energy band (3-10 keV). When a self-consistent model is applied to the whole broad-band data, the observed reflection component appears to be entirely associated with the relativistic component of the iron $\mathrm{K} \alpha$ line.

Conclusions. The implied scenario, though strongly model-dependent, requires that all the reprocessing spectral components from Compton-thick material be associated with the accretion disc, and no evidence of a classical pc-scale torus is found. The narrow core of the neutral iron $\mathrm{K} \alpha$ line is therefore produced in Compton-thin material, such as the BLR, similarly to what is found in another Seyfert galaxy, NGC 7213, but with the notable difference that MCG+8-11-11 presents spectral signatures from an accretion disc. The very low accretion rate of NGC 7213 could explain the lack of relativistic signatures in its spectrum, but the absence of the torus in both sources is more difficult to explain, since their luminosities are comparable, and their accretion rates are completely different.
\end{abstract}

Key words. galaxies: active - galaxies: Seyfert - X-rays: general

\section{Introduction}

The X-ray spectrum of unobscured AGN is characterised by ubiquitous and, generally, non-varying features, such as the neutral iron $\mathrm{K} \alpha$ narrow core and the Compton reflection component (e.g., Perola et al. 2002; Bianchi et al. 2007), whose origin appears quite clearly established within the framework of standard unification models as the pc-scale "torus" (Antonucci 1993). On the other hand, there are other spectral components, such as the warm absorber, the soft excess, and the relativistic component of the iron $\mathrm{K} \alpha$ line, which are present in only a fraction of the sources, and may vary in different observations of the same object (e.g. Piconcelli et al. 2005; Nandra et al. 2007). Their origin is less clear, and, as in the case of the relativistic iron line, may pose some crucial problems to the standard views.

$\mathrm{X}$-ray emission from the accretion disc, as a result of the reprocessing of the $\mathrm{X}$-ray photons produced in the corona, is a fundamental component of any AGN model, and should be characterised by a strong iron $\mathrm{K} \alpha$ line, whose profile can be clearly distinguished from the narrow core, because of wellknown relativistic effects occurring near the black hole (see e.g.
Fabian et al. 2000). However, this relativistic profile, when large samples of AGN are systematically analysed, is not observed in all the sources (see e.g. Nandra et al. 2007; Longinotti et al. 2008). Although at least a part of this problem must be related to the signal-to-noise ratio of the available X-ray spectra (indeed the fraction of sources with relativistic signatures appears to be larger when only sufficiently exposed X-ray spectra are analysed: see, e.g., de la Calle Pérez et al. 2010), the relativistic component of the iron $\mathrm{K} \alpha$ line still appears not to be ubiquitous, and, even when present, its equivalent width $(E W)$ is often lower than expected.

These results imply that a revision of the standard models of accretion discs is required. Truncation of the disc, a highly ionization state, and complex illumination may all be invoked to explain the observed deviations from the current picture, but it is most fundamental to understand the ultimate drivers of this behaviour, such as the accretion rate, the luminosity, and the black hole $(\mathrm{BH})$ mass. In this respect, evidence is mounting that other ingredients of the unification models may behave differently from expected, and be closely related to the above-mentioned physical parameters. For example, the broad line region (BLR) 
was found to be most likely absent in some sources (e.g. Bianchi et al. 2008a; Panessa et al. 2009), to act as a Compton-thick X-ray absorber in others (e.g. Elvis et al. 2004; Risaliti et al. 2005; Puccetti et al. 2007; Bianchi et al. 2009b), or even to produce most of the observed neutral iron $\mathrm{K} \alpha$ line, when no other evidence of the torus could be found in the data (Bianchi et al. 2008b).

MCG+8-11-11 $(z=0.0205)$ is one of the brightest AGN in the X-ray band, detected by INTEGRAL with a $F_{20-100 \mathrm{keV}} \simeq$ $1.2 \times 10^{-10}$ cgs (Beckmann et al. 2009). ASCA (Grandi et al. 1998) and BeppoSAX (Perola et al. 2000, 2002) found that the spectrum is well fitted by a pretty standard model composed of a power law, a warm absorber, a Compton reflection component, and an iron $\mathrm{K} \alpha$ line. The higher quality of the XMM-Newton spectrum (Matt et al. 2006) revealed some interesting features: the lack of a soft excess, a large reflection component, and a narrow iron line with a low $E W$. The presence of a large, relativistically broadened line arising from the accretion disk could also be excluded in these data.

In this paper, we present a long (100 ks) Suzaku observation of MCG+8-11-11. In addition to its 54-month Swift BAT spectrum, we explore different energy bands, in order to understand the origin of the X-ray spectral components of this bright Seyfert 1 . The availability of a high signal-to-noise spectrum in such a broad band (0.6-150 keV) provides us with the opportunity to study in detail all the contributions from the materials invoked in unification models, and infer their presence/absence and general properties.

\section{Data reduction}

MCG+8-11-11 was observed by Suzaku on 2007, September 17, for $100 \mathrm{ks}$ (OBSID 702112010). X-ray Imaging Spectrometer (XIS) and Hard X-ray Detector (HXD) event files were reprocessed with the latest calibration files available (2010-03-23 release), using fTOOLS 6.9 and SuZAKU software Version 16, adopting standard filtering procedures. Source and background spectra for all the three XIS detectors were extracted from circular regions with radius of 250 pixels ( $\simeq 260 \mathrm{arcsec}$ ), avoiding the calibration sources. Response matrices and ancillary response files were generated using XISRMFGEN and XISSIMARFGEN. We downloaded the tuned non-X-ray background (NXB) for our $\mathrm{HXD} / \mathrm{PIN}$ data provided by the HXD team and extracted source and background spectra using the same good time intervals. The PIN spectrum was then corrected for dead time, and the exposure time of the background spectrum was increased by a factor of 10, as required. Finally, the contribution from the cosmic $\mathrm{X}$-ray background (CXB) was subtracted from the source spectrum, simulating it as suggested by the HXD team.

We extracted the light curves of the three XIS in the soft and the hard energy band (see Fig. 1 for the XISO). The source exhibits some variability in both bands, but the hardness ratio is practically constant within $10 \%$. On the other hand, the HXD pin light curve does not show any significant evidence of variability. Therefore, we decided to analyse the total spectra, without any additional timing analysis.

The front-illuminated XIS0 and XIS3 were fitted between 0.6 and $10 \mathrm{keV}$, while the back-illuminated XIS1 was used up to $8 \mathrm{keV}$, because the background above that energy becomes significant with respect to the source spectrum for that instrument. The band 1.6-2.1 keV was excluded from all the fits, because of known inter-calibration issues at those energies due to the $\mathrm{Si}$ $\mathrm{K}$ edge. The normalizations of XIS1 and XIS3 with respect to XIS0 were left free in the fits, and always resulted in agreement



Fig. 1. Soft (0.3-2 keV) and hard (2-10 keV) light-curves of the Suzaku 100 ks observation of MCG+8-11-11 (only the XIS0 is plotted for clarity). The bottom panel shows the ratio between the two curves.

to within $2 \%$. A constant factor of 1.18 was used instead between the PIN and the XIS0, as recommended for observations taken in the HXD nominal position. In the following, all the PIN fluxes are given with respect to the XIS0 flux scale, which is 1.18 times lower than the HXD absolute flux scale.

In the following, errors correspond to the $90 \%$ confidence level for one interesting parameter $\left(\Delta \chi^{2}=2.71\right)$, unless not otherwise stated. The adopted cosmological parameters are $H_{0}=$ $70 \mathrm{~km} \mathrm{~s}^{-1} \mathrm{Mpc}^{-1}, \Omega_{\Lambda}=0.73$, and $\Omega_{\mathrm{m}}=0.27$ (i.e., the default ones in xspec 12.6.0: Arnaud 1996). We use the Anders \& Grevesse (1989) abundances and the photoelectric absorption cross-sections by Balucinska-Church \& McCammon (1992).

\section{Data analysis}

\subsection{The iron line complex}

As a first step, we fitted the $3-10 \mathrm{keV}$ spectra with a simple power law. The photon index is quite flat $(1.62 \pm 0.02)$, and clear residuals remain between 6 and $7 \mathrm{keV}$ (see Fig. 2). Three Gaussian lines are required by the data, at $6.401 \pm 0.008$, $6.66 \pm 0.06$, and $6.96 \pm 0.02 \mathrm{keV}$, that are consistent with emission from neutral, He-like, and $\mathrm{H}$-like $\mathrm{Fe} \mathrm{K} \alpha$. An absorption edge from neutral iron (its energy fixed to $7.11 \mathrm{keV}$ : Bearden 1967) is also marginally detected, with an optical depth $\tau=0.04 \pm 0.02$ (model I). On the other hand, a Fe $\mathrm{K} \beta$ emission line is not required by the data, with an upper limit of $\simeq 7 \%$ with respect to the flux of the $\mathrm{Fe} \mathrm{K} \alpha$. In an analogous way, the $\mathrm{K} \alpha$ emission from nickel is not detected. While the two ionised iron lines are consistent with being unresolved $(\sigma<80 \mathrm{eV})$, the neutral Fe $\mathrm{K} \alpha$ is resolved, with $\sigma=52_{-19}^{+17} \mathrm{eV}$. This width is larger than the calibration uncertainties in the XIS redistribution matrix ${ }^{1}$, and corresponds to a full width at half maximum $F W H M=6500 \pm 1900 \mathrm{~km} \mathrm{~s}^{-1}$, if caused by Doppler broadening.

\footnotetext{
1 We extracted the calibration spectra for this observation, and fitted them separately for each XIS with a model consisting of three emission lines: the Mn K $\alpha$ doublet (the separation of the lines fixed to be $0.0111 \mathrm{keV}$ : Bearden 1967) and the $\mathrm{K} \beta$. The width of the three lines were kept linked. We recovered upper limits in all the spectra: $23 \mathrm{eV}$ (XIS0), 25 eV (XIS1), 14 eV (XIS3).
} 

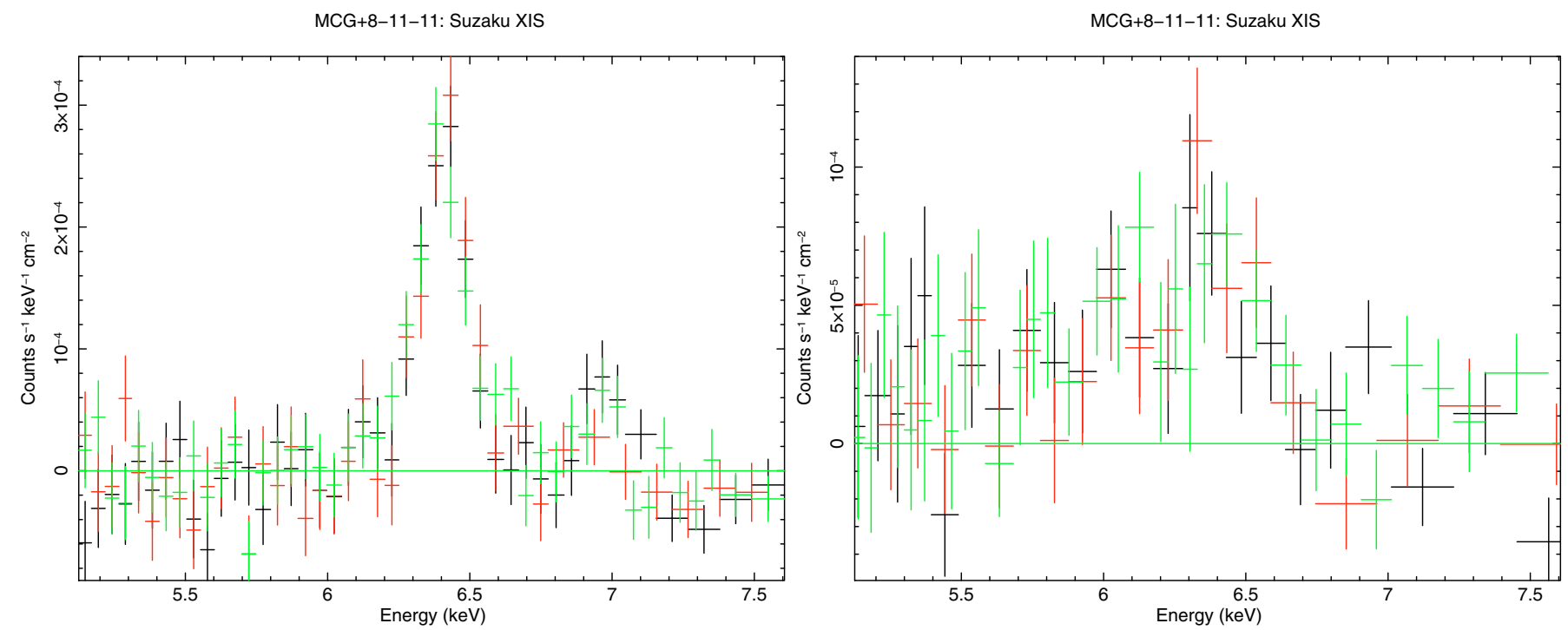

Fig. 2. Left: residuals in the (rest-frame) iron line band, after a 3-10 keV fit on the three Suzaku XIS spectra, with a simple power law. Right: the same as above, but for model III, after the relativistic component of the iron line is removed (see Table 1). Data have been rebinned for clarity.

The neutral iron $\mathrm{K} \alpha$ width can be explained by the contribution of a Compton shoulder (CS), as suggested by some residuals redwards of the line. We therefore added another Gaussian line with centroid at $6.3 \mathrm{keV}$ and $\sigma=40 \mathrm{eV}$ (both fixed: see Matt 2002). The improvement of the fit is marginal (model II: $\left.\Delta \chi^{2}=4\right)$, but the Fe $\mathrm{K} \alpha$ line is now unresolved $(\sigma<45 \mathrm{eV})$. However, its centroid is shifted to $6.421_{-0.012}^{+0.011} \mathrm{keV}$, and the flux ratio of the CS to the core is $32_{-14}^{+10} \%$, which is larger than the theoretical expectations for Compton-thick matter (Matt 2002).

The width of the iron line and the redwards residuals may alternatively be signatures of a mildly relativistic line. We tested this scenario by adding to the unresolved core a KYRLINE model component (Dovčiak et al. 2004), adopting a fixed centroid energy at $6.4 \mathrm{keV}$, an outer radius of $400 r_{\mathrm{g}}$, and a power-law in$\operatorname{dex} \beta=3$ for the radial dependence of the emissivity. This fit (model III) is statistically preferred to the others $\left(\Delta \chi^{2}=15\right.$ and 11 with respect to models I and II, and a null hypothesis probability of 0.43 , which is significantly better than 0.22 and 0.26 , respectively). The inner radius $\left(<20 r_{\mathrm{g}}\right)$ and the inclination angle $(\theta=30 \pm 3 \mathrm{deg})$ can be constrained in the fit, while the $\mathrm{BH}$ spin is completely unconstrained, and therefore fixed to be zero. The FexXV line is no longer required by the data: if the centroid energy is allowed to vary between or be fixed to the theoretical values for each component of this multiplet (see e.g. Bianchi et al. 2005), only upper limits are recovered (of the order of $\left.1 \times 10^{-5} \mathrm{ph} \mathrm{cm}^{-2} \mathrm{~s}^{-1}\right)$. The iron $\mathrm{K}$ edge is also an upper limit in this model $(\tau<0.03)$. The final fit is very good $\left(\chi^{2}=301 / 298\right.$ d.o.f.), and is applied in the following broadband fits, where the presence of the relativistic component is investigated again once a more sophisticated model for the continuum is adopted. Table 1 summarizes the best-fit parameters for the models described in this section.

\subsection{The $0.6-10 \mathrm{keV}$ band}

The best fit adopted for the 3-10 keV band (model III in Table 1) can be applied to the whole XIS band, once Galactic absorption along the line of sight is taken into account. We recover
Table 1. Best-fit model parameters for the emission features in the Suzaku XIS spectra (3-10 keV).

\begin{tabular}{|c|c|c|c|c|}
\hline & & I & $\overline{\mathrm{III}}$ & $\overline{\text { IIII }}$ \\
\hline $\mathrm{Fe} \mathrm{K} \alpha$ & $\begin{array}{c}E_{0} \\
\sigma \\
F \\
E W\end{array}$ & $\begin{array}{c}6.401 \pm 0.008 \\
52_{-19}^{+17} \\
6.6 \pm 0.6 \\
89 \pm 8\end{array}$ & $\begin{array}{c}6.421_{-0.012}^{+0.011} \\
<45 \\
5.0 \pm 0.5 \\
67 \pm 7\end{array}$ & $\begin{array}{c}6.396_{-0.004}^{+0.016} \\
0^{*} \\
4.9 \pm 0.5 \\
67 \pm 7\end{array}$ \\
\hline $\mathrm{Fe} \mathrm{K} \alpha \mathrm{CS}$ & $F$ & - & $1.6_{-0.7}^{+0.5}$ & - \\
\hline $\mathrm{Fe} \mathrm{XXV} \mathrm{K} \alpha$ & $\begin{array}{c}E_{0} \\
F \\
E W\end{array}$ & $\begin{array}{c}6.65 \pm 0.07 \\
0.6 \pm 0.4 \\
8 \pm 5\end{array}$ & $\begin{array}{c}6.63_{-0.07}^{+0.05} \\
0.8_{-0.3}^{+0.4} \\
11_{-4}^{+6}\end{array}$ & $\begin{array}{l}- \\
- \\
-\end{array}$ \\
\hline Fe XXVI K $\alpha$ & $\begin{array}{c}E_{0} \\
F \\
E W\end{array}$ & $\begin{array}{c}6.97 \pm 0.03 \\
1.5 \pm 0.4 \\
23 \pm 6\end{array}$ & $\begin{array}{c}6.970_{-0.032}^{+0.018} \\
1.5 \pm 0.3 \\
24 \pm 5\end{array}$ & $\begin{array}{c}6.960_{-0.022}^{+0.019} \\
1.9 \pm 0.4 \\
29 \pm 6\end{array}$ \\
\hline $\mathrm{Fe} \mathrm{K} \alpha_{k y}$ & $\begin{array}{c}\theta \\
r_{i} \\
F \\
E W\end{array}$ & $\begin{array}{l}- \\
- \\
- \\
-\end{array}$ & $\begin{array}{l}- \\
- \\
- \\
-\end{array}$ & $\begin{array}{c}30 \pm 3^{\circ} \\
<20 \\
6.1_{-1.5}^{+1.7} \\
90 \pm 20\end{array}$ \\
\hline Fe K edge & $\begin{array}{c}\tau \\
\chi^{2} / \text { d.o.f. }\end{array}$ & $\begin{array}{c}0.04 \pm 0.02 \\
316 / 297\end{array}$ & $\begin{array}{c}0.04 \pm 0.02 \\
312 / 297\end{array}$ & $\begin{array}{c}<0.03 \\
301 / 298\end{array}$ \\
\hline
\end{tabular}

Notes. The three models are described in the text. Energies $\left(E_{0}\right)$ are in $\mathrm{keV}, E W \mathrm{~s}$ and $\sigma$ in $\mathrm{eV}$, fluxes $(F)$ in units of $10^{-5} \mathrm{ph} \mathrm{cm}^{-2} \mathrm{~s}^{-1}$, and radii $\left(r_{i}\right)$ in $r_{\mathrm{g}}$.

a neutral column density ${ }^{2}$ of $\simeq 2.1-2.4 \times 10^{21} \mathrm{~cm}^{-2}$, depending on the adopted complete model (see below and Table 2). This measure is in good agreement with the estimate of Dickey \& Lockman (1990), $2.09 \times 10^{21} \mathrm{~cm}^{-2}$, but somewhat larger than the estimate of Kalberla et al. (2005), $1.76 \times 10^{21} \mathrm{~cm}^{-2}$. Fixing

2 This value of column density was found by adopting the TBABS model, and the abundances and cross-sections minimizing the $\chi^{2}$ (see Sect. 2 for the final choice). 




Fig. 3. Best-fit model and $\Delta \chi^{2}$ for the three XIS spectra, with the same model as in the $3-10 \mathrm{keV}$ fit, but including Galactic absorption, a warm absorber and an unidentified absorption edge (see text for details). Note the residuals above $8 \mathrm{keV}$.

the Galactic column density to the latter value, a comparable $\chi^{2}$ is recovered by adding an additional neutral column density at the redshift of the source $\left(4.6_{-1.4}^{+0.9} \times 10^{20} \mathrm{~cm}^{-2}\right)$, without affecting any other parameter of the fit.

However, there is clearly some curvature left in the residuals at low energies, resulting in a $\chi^{2}=681 / 515$. Since additional neutral absorption at the redshift of the source is not required by the data, we added a warm absorber component (at first we adopted ABSORI, with the temperature of the material being kept fixed to $10^{6} \mathrm{~K}$, which can be directly compared to the results reported by Matt et al. 2006), improving our fit down to $\chi^{2}=641 / 513$, by using best-fit model parameters for the warm absorber in good agreement with those found by Matt et al. (2006). As already noted by these authors, a single-zone absorber cannot properly describe the data. Some residuals remain around $0.8 \mathrm{keV}$ : they can be fitted by an edge, found at $0.82_{-0.03}^{+0.02} \mathrm{keV}$, with $\tau=0.050 \pm 0.017$. The energy of the edge is intermediate between $\mathrm{K}$ absorption from O VII $(0.7393 \mathrm{keV})$ and O VIII $(0.8714 \mathrm{keV})$, but inconsistent with both. We note here that the energy of this feature is not even close to the absorption features observed in the XMM-Newton RGS spectra (Matt et al. 2006).

The resulting $\chi^{2}=616 / 511$ would be acceptable, since most of the residuals appears to be caused mainly by poor background subtraction (such as between 7 and $8 \mathrm{keV}$ for the XIS0) and some inter-calibration issues between the instruments. However, above $8 \mathrm{keV}$ the data-points appears to be systematically higher than the model (see Fig. 3). We therefore added a Compton reflection component (model PEXRAV, with inclination angle fixed to $30^{\circ}$ and cutoff energy to $150 \mathrm{keV}$, see next section), which indeed improves the fit, for a final value of $\chi^{2}=586 / 509$. The photon index is now steeper $(\Gamma=1.772 \pm 0.017)$ than the one found in the previous section for the limited band, and the amount of Compton reflection is $R=0.9 \pm 0.3$, with an iron abundance of $A_{\mathrm{Fe}}=0.6_{-0.2}^{+0.3}$. The need for a relativistic component of the neutral iron line is far less significant, given the large inner radius $\left(110_{-80}^{+180} r_{\mathrm{g}}\right)$ and the small $E W(30 \pm 12 \mathrm{eV})$. To understand whether the disappearance of the relativistic line and the other fitting parameters depend strongly on our choice of a simple model for warm absorption, we tried a more sophisticated warm absorber model with respect to ABSORI, which only takes into account absorption edges.

We prepared an ad-hoc table produced with the photoionization code CLOUDY C08.00 (Ferland et al. 1998). The model ingredients are: (1) a multi-component continuum mimicking a typical AGN spectral energy distribution (SED), with $\alpha_{o x}=1.26$ and $\Gamma_{2-10 \mathrm{keV}}=1.8$, as extracted from the results of the simultaneous UV and X-ray data of the XMM-Newton observation of MCG+8-11-11 (Matt et al. 2006); (2) constant electron density $^{3} n_{\mathrm{e}}=10^{4} \mathrm{~cm}^{-3}$; (3) ionization parameter ${ }^{4}$ in the range $\log U=-2.0: 2.5$; (4) intervening column density in the range $\log N_{N H}=20.0: 22.0$. The resulting fit is marginally better than the one found with ABSORI $\left(\Delta \chi^{2}=5\right)$ and we note that the unidentified absorption edge is still required by the data, with parameters consistent with those found in the other fit. However, the properties of the warm absorber gas are quite different, both the column density and the ionization parameter being significantly lower in the CLOUDY best fit, confirming that the signatures of warm absorption are weak in this source.

All the other best-fit parameters are indeed consistent with those found with ABSORI, including the narrowish and weak relativistic component of the $\mathrm{Fe} \mathrm{K} \alpha$ emission line. The fit is improved neither when a velocity shift is allowed for the warm absorbing gas, nor with the addition of a second warm absorber component. The first two columns of Table 2 summarize and compare the results of these two fits.

\subsection{The broad-band fit}

When the best-fit model found for the 0.6-10 keV XIS spectra is extrapolated to higher energies, the PIN data points all fall well below the expectations (see Fig. 4). Allowing the model to be adjusted for the high-energy data, the best-fit model is acceptable $\left(\chi^{2}=625 / 524\right)$, the main difference with the limited band fit being the very low value of the Compton reflection component $R=0.13_{-0.06}^{+0.08}$. The relativistic component of the iron $\mathrm{K} \alpha$ line is now very significant, recovering the same properties found in model III of the $3-10 \mathrm{keV}$ fit (see Sect. 3.1 and Table 1). The cutoff energy cannot be measured, with a $99 \%$ lower limit of $\simeq 130 \mathrm{keV}$ (for two interesting parameters, when we assume the reflection amount to be the second interesting parameter).

To constrain the cutoff energy, we then added the Swift BAT spectrum (in the range 15-150 keV), from the 54-month source catalog (Cusumano et al., A\&A submitted). The normalization factor between the XIS0 and the BAT spectrum was allowed to vary, to take into account source variability during the 54 months of integration. The best-fit value of this factor $(0.76 \pm 0.03)$ indeed clearly suggests that the Suzaku observation caught the source in a flux state significantly higher than the average of the BAT campaign. This is confirmed by the BAT lightcurve, which show significant variability in the 54 months, and a flux comparable to the one measured by the PIN at the date of the Suzaku observation (see Fig. 5). After assuming that this flux variability does not imply a spectral variability, we refitted the best-fit model including the BAT spectrum, and found that $E_{\mathrm{c}}=150_{-20}^{+30} \mathrm{keV}$. This value, which remains consistent with the

\footnotetext{
${ }_{3}$ Models with higher electron densities up to $n_{\mathrm{e}}=10^{9} \mathrm{~cm}^{-3}$ have also been produced, but all the following fits were found to be insensitive to this parameter, as expected (see e.g. Nicastro et al. 1999).

${ }^{4}$ We use the standard definition $U=\frac{\int_{v_{R}}^{\infty} \frac{L_{v}}{h v} \mathrm{~d} v}{4 \pi r^{2} c n_{\mathrm{e}}}$ where $c$ is the speed of light, $r$ the distance of the gas from the illuminating source, $n_{\mathrm{e}}$ the electron density, and $v_{R}$ the frequency corresponding to 1 Rydberg (Osterbrock \& Ferland 2006).
} 

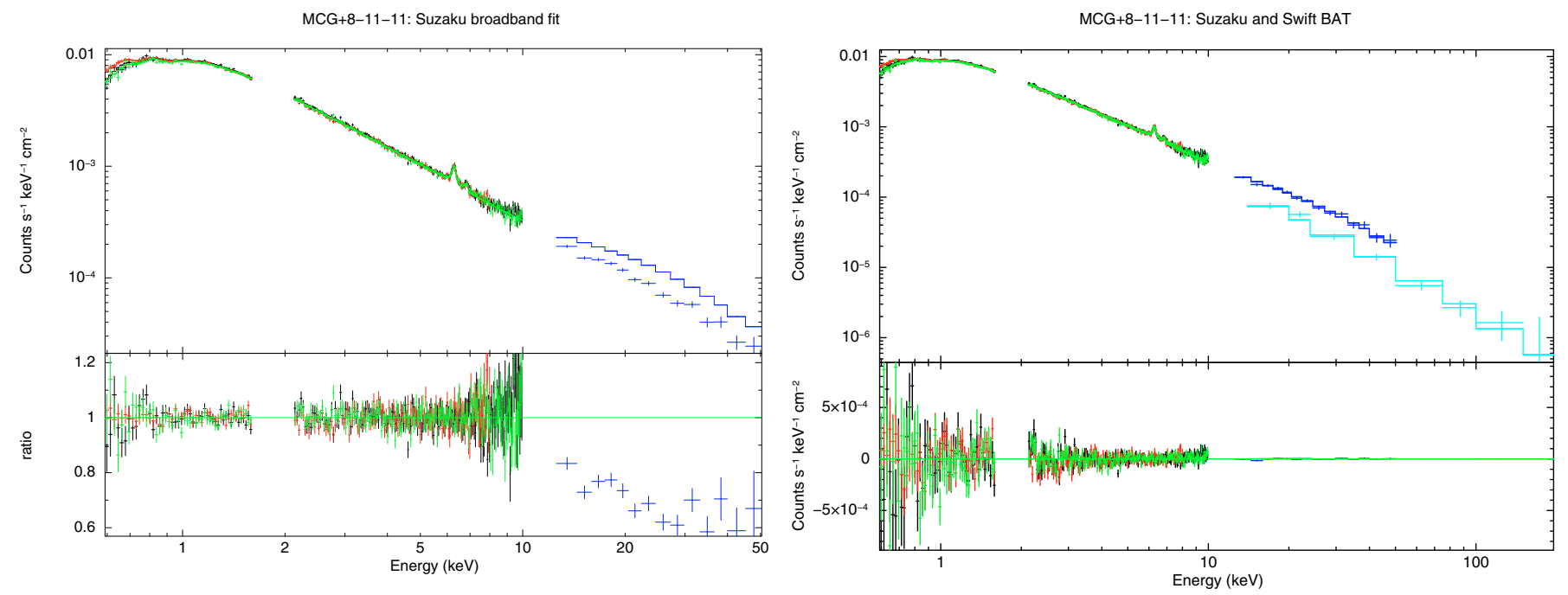

Fig. 4. Left: the best-fit model for the $0.6-10 \mathrm{keV}$ band, when extrapolated to the band covered by the PIN spectrum. Right: the Suzaku+Swift BAT best-fit model in the whole $0.6-150 \mathrm{keV}$ band.

Table 2. Best-fits for the Suzaku XIS spectra (0.6-10 keV, first two columns) and the Suzaku+Swift BAT spectra (0.6-150 keV, last two columns).

\begin{tabular}{lcccc}
\hline \hline & \multicolumn{2}{c}{$0.6-10 \mathrm{keV}$} & \multicolumn{2}{c}{$0.6-150 \mathrm{keV}$} \\
& ABSORI & CLOUDY & ABSORI & CLOUDY \\
\hline$N_{\mathrm{Hg}}\left(10^{21} \mathrm{~cm}^{-2}\right)$ & $2.37 \pm 0.05$ & $2.22_{-0.15}^{+0.09}$ & $2.32 \pm 0.04$ & $2.18_{-0.11}^{+0.08}$ \\
$N_{\mathrm{Hw}}\left(10^{22} \mathrm{~cm}^{-2}\right)$ & $0.9_{-0.6}^{+1.1}$ & - & $1.5_{-0.6}^{+1.0}$ & - \\
$\log N_{\mathrm{Hw}}$ & - & $20.7 \pm 0.2$ & - & $20.5_{-0.5}^{+0.3}$ \\
$\xi\left(\mathrm{erg} \mathrm{s}^{-1} \mathrm{~cm}\right)$ & $600_{-200}^{+900}$ & - & $600_{-160}^{+370}$ & - \\
$\log U$ & - & $-1.2 \pm 0.6$ & - & $-1.1_{-0.8}^{+1.1}$ \\
$E_{\text {edge }}$ & $0.827_{-0.017}^{+0.015}$ & $0.829 \pm 0.015$ & $0.82 \pm 0.02$ & $0.83 \pm 0.02$ \\
$\tau_{\text {edge }}$ & $0.072 \pm 0.018$ & $0.074_{-0.020}^{+0.019}$ & $0.060 \pm 0.017$ & $0.060 \pm 0.018$ \\
$\Gamma$ & $1.772 \pm 0.017$ & $1.80 \pm 0.02$ & $1.740 \pm 0.012$ & $1.743 \pm 0.012$ \\
$R$ & $0.9 \pm 0.3$ & $1.4 \pm 0.3$ & $0.23 \pm 0.04$ & $0.27 \pm 0.05$ \\
$E_{\mathrm{c}}$ & $150^{*}$ & $150^{*}$ & $150_{-20}^{+30}$ & $150_{-20}^{+30}$ \\
$A_{\mathrm{Fe}}$ & $0.6_{-0.2}^{+0.3}$ & $0.73_{-0.18}^{+0.21}$ & $<0.33$ & $<0.15$ \\
$F_{0.5-2}$ & $2.15 \pm 0.02$ & $2.157 \pm 0.018$ & $2.151 \pm 0.017$ & $2.14 \pm 0.03$ \\
$F_{2-10}$ & $6.66 \pm 0.04$ & $6.66 \pm 0.03$ & $6.61 \pm 0.03$ & $6.60 \pm 0.03$ \\
$F_{15-50}$ & - & - & $7.9 \pm 0.3$ & $8.1 \pm 0.2$ \\
$L_{0.5-2}$ & $3.73 \pm 0.03$ & $3.59 \pm 0.03$ & $3.70 \pm 0.03$ & $3.64 \pm 0.05$ \\
$L_{2-10}$ & $6.45 \pm 0.04$ & $6.41 \pm 0.03$ & $6.46 \pm 0.03$ & $6.38 \pm 0.03$ \\
$L_{15-50}$ & - & - & $6.4 \pm 0.2$ & $6.48 \pm 0.16$ \\
$\chi^{2} /$ d.o.f. & $586 / 509$ & $581 / 509$ & $640 / 531$ & $658 / 531$ \\
\hline
\end{tabular}

Notes. The adopted model is the same in all the cases: TBABS*ZEDGE*ABSORI*(KYRLINE+ZGAUSS+ZGAUSS+PEXRAV in XSPEC jargon (see also model III in Table 1). For each band, results are reported when the warm absorber is modelled with ABSORI and CLOUDY. See text for details. Fluxes are in $10^{-11} \mathrm{erg} \mathrm{cm}^{-2} \mathrm{~s}^{-1}$, unabsorbed luminosities in $10^{43} \mathrm{erg} \mathrm{s}^{-1} .15-50 \mathrm{keV}$ fluxes and luminosities are relative to the XIS normalization (a factor of 1.18 lower than the HXD absolute flux).

lower limit found with the Suzaku data only, is also in agreement with that measured by BeppoSAX $\left(170_{-80}^{+300} \mathrm{keV}\right.$ : Perola et al. $2000)$ and only marginally inconsistent with $O S S E\left(270_{-70}^{+90} \mathrm{keV}\right.$ : Grandi et al. 1998).

In contrast to the $0.6-10 \mathrm{keV}$ fit discussed in the previous section, the best-fit model for the broad-band 0.6-150 keV spectra is achieved with the warm absorber parametrised by ABSORI: a significantly poorer fit is found when the CLOUDY table is used $\left(\Delta \chi^{2}=18\right.$ : see Table 2). In any case, all the other parameters appears to be independent of the choice of the warm absorber. In particular, both fits recover a low reflection component $(R \simeq 0.2-0.3)$ and an iron abundance significantly lower than the solar one. Their inter-correlation and dependence upon other relevant parameters are shown in Fig. 6. The relativistic component of the iron line is now interestingly very significant, and its best-fit model parameters are similar to those found in our first phenomenological fits of the iron line complex (see Sect. 3.1 and Table 1).

The broad-band best fit is not as statistically good as the one constrained by the XIS band-pass, being $\chi^{2}=640 / 531$. Most of the residuals are due to the XIS data-points above $8 \mathrm{keV}$, which are on average higher than the model: as we have seen in the previous section, they were most likely the main reasons for requiring an high Compton reflection in the $0.6-10 \mathrm{keV}$ band, but they are apparently inconsistent with the PIN flux. We alternatively tried to keep the iron abundance fixed to the solar value, leaving the inclination angle of the reflecting material free in the fit. The resulting $\chi^{2}$ is comparable to the best fit $\left(\chi^{2}=646 / 531\right)$ with a 


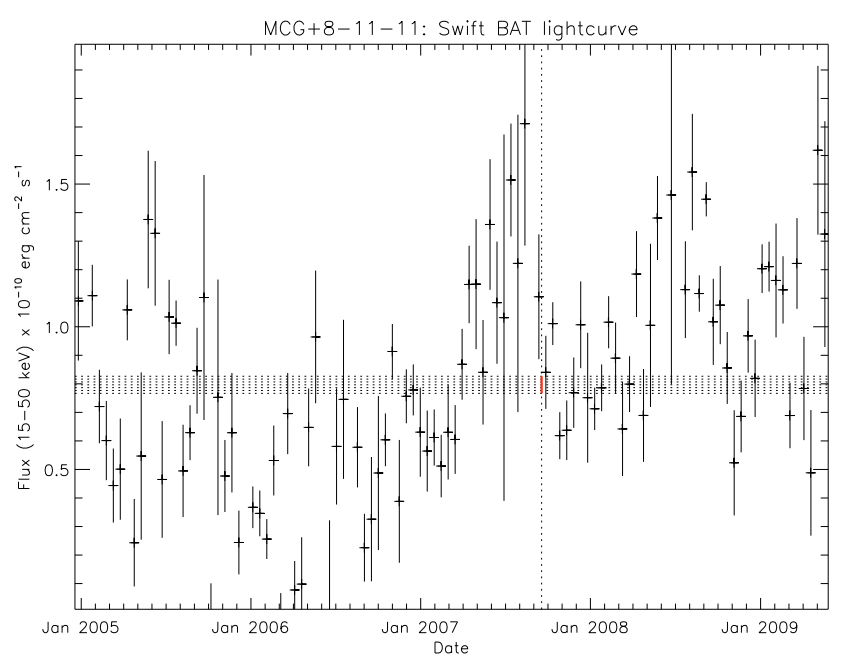

Fig. 5. The 15-50 keV Swift BAT 54-month light curve of MCG+8-1111 (a bin is 15 days). The countrate was converted to a $15-50 \mathrm{keV}$ flux, assuming no spectral variation. The Suzaku PIN flux is shown in comparison, with its uncertainty, with the dotted lines and the red symbol. The agreement at the date of the Suzaku observation is good.

very high inclination angle $(\cos i<0.30)$. The reflection fraction is now much larger, but loosely constrained $\left(R=2.9_{-2.2}^{+0.6}\right)$.

\section{Discussion}

\subsection{Comparison with previous observations}

In the past 15 years, MCG+8-11-11 has been observed in the $\mathrm{X}$-rays by $A S C A$ (simultaneously with OSSE, Grandi et al. 1998), BeppoSAX (Perola et al. 2000), and XMM-Newton (Matt et al. 2006). Despite significant flux variability (a factor $\simeq 3$ between $A S C A$ and the present Suzaku observation), there is no clear evidence of variations of the spectral shape of the source. The photon index of the primary continuum was also measured in the range 1.7-1.8, a difference of 0.05 arising in this $S u z a k u$ observation whether the PIN data are considered or not in the fit. The warm absorber, if modelled in the same way (i.e. ABSORI) also gives consistent results between XMM-Newton and Suzaku. The notable absence of a soft excess is confirmed in all the observations, even if the large Galactic column density may help to conceal it in the data.

The iron $\mathrm{K} \alpha$ neutral line has a complex profile, and is probably composed of different components. Both ASCA and BeppoSAX data suggested a broadish profile $(\sigma \simeq 0.2-0.3 \mathrm{keV}$, but marginally resolved) with $E W$ s consistent with a constant line flux (Grandi et al. 1998; Perola et al. 2000). The same applies to the XMM-Newton and the Suzaku iron $\mathrm{K} \alpha$ flux, once all the components required by the data are taken into account. However, while these results suggest that the iron $\mathrm{K} \alpha$ flux did not vary significantly, the errors and the differences in modelling its profile make it difficult to extract any other useful information from this apparent lack of response to the primary flux variability.

As for the Compton reflection component, all previous observations are consistent with values around $R \simeq 1-1.5$, but with a good (statistical) constraint only on the XMM-Newton data (Matt et al. 2006). The Suzaku best-fit model limited to the $0.6-10 \mathrm{keV}$ band is consistent with these values, but still in agreement, within the errors, with a scenario where the reflection component flux is constant, and the value of $R$ anticorrelates with the flux of the primary continuum, as expected if the reprocessing originates in a distant material, such as the torus. However, when the PIN data are taken into account, the Compton reflection drops to a significantly lower value, around $0.2-0.3$ (see Table 2). This is also supported by the preference for very low iron abundance and/or large inclination angles with respect to the line-of-sight, probably caused by the iron edge being not particularly deep, as confirmed by the phenomenological fit we performed in the 3-10 keV band (see Sect. 3.1), where the edge is significant only when the relativistic component of the iron $\mathrm{K} \alpha$ line is not included in the model. We note that an iron abundance lower than solar is also preferred in the $0.6-10 \mathrm{keV}$ fit, as found by Matt et al. (2006) in the XMM-Newton data. In these cases, though, the relativistic component of the iron $\mathrm{K} \alpha$ line is completely negligible.

\subsection{A global scenario}

To test the data with a self-consistent global scenario, we replaced in the model described in Sect. 3.3 (see also Table 2) the PEXRAV and the narrow core of the iron $\mathrm{K} \alpha$ line with the PEXMON model (Nandra et al. 2007), which includes at the same time the incident primary power law, and all the reprocessing components produced by Compton-thick material: the emission features (Fe $\mathrm{K} \alpha, \mathrm{K} \beta$ and Compton shoulder, and the Ni K $\alpha$ emission line) and the Compton reflection hump, with all the relevant inter-dependencies (inclination, index, and abundance). The resulting fit is worse than the best fit, but still acceptable $\left(\chi^{2}=650 / 532\right)$. The iron abundance is now much higher $\left(A_{\mathrm{Fe}}=1.1_{-0.2}^{+0.3}\right)$, and the amount of reflection is somewhat higher ( $\left.R=0.34_{-0.04}^{+0.05}\right)$. This is due to the need to model the narrow component of the iron neutral $\mathrm{K} \alpha$ line self-consistenly with the Compton reflection hump. On the other hand, all the other parameters are consistent with those found in our best fit presented in the previous section, within the errors, including the warm absorber (ABSORI) and the absorption edge.

We then tested whether an additional component of the neutral iron line narrow core is needed by the data. A possibility is a contribution from the BLR. In the case of MCG+8-11-11, the $F W H M$ of the $\mathrm{H} \alpha$ is $2920 \mathrm{~km} \mathrm{~s}^{-1}$, and that of the $\mathrm{H} \beta 3630 \mathrm{~km} \mathrm{~s}^{-1}$ (Osterbrock \& Shuder 1982). We therefore added a neutral Fe $\mathrm{K} \alpha$ line with $\sigma$ fixed at $30 \mathrm{eV}$ (consistent with a production in the same gas in which $\mathrm{H} \alpha$ and $\mathrm{H} \beta$ originate), and fixed the iron abundance at the Solar value. The fit only slightly improves $\left(\chi^{2}=647 / 532\right)$, due to the marginal significance of the iron $\mathrm{K} \alpha$ line supposedly produced in the BLR $(E W=14 \pm 12 \mathrm{eV})$. This test appears to suggest that the observed iron $\mathrm{K} \alpha$ line can be satisfactorily accompanied by the measured low reflection component, in a self-consistent way. But this is due to only the narrow core needing to be modelled, with $E W=60-70 \mathrm{eV}$ (see Table 2).

Indeed, in all these fits, a significant contribution from a relativistic component to the iron line is still required. This component should be accompanied by its own Compton reflection, produced in the accretion disc. To test this scenario, we therefore added another PEXMON component (only the reprocessing components), convolved with a relativistic kernel (KDBLUR) to model the relativistic effects occurring in the inner accretion disc, close to the BH. The final best-fit model improves, and it is almost statistically equivalent to the best fit found in Sect. 3.3 $\left(\chi^{2}=644 / 532\right)$. All the reflection component originates in the disc, with $R=0.31_{-0.14}^{+0.03}$, while the contribution from the torus 

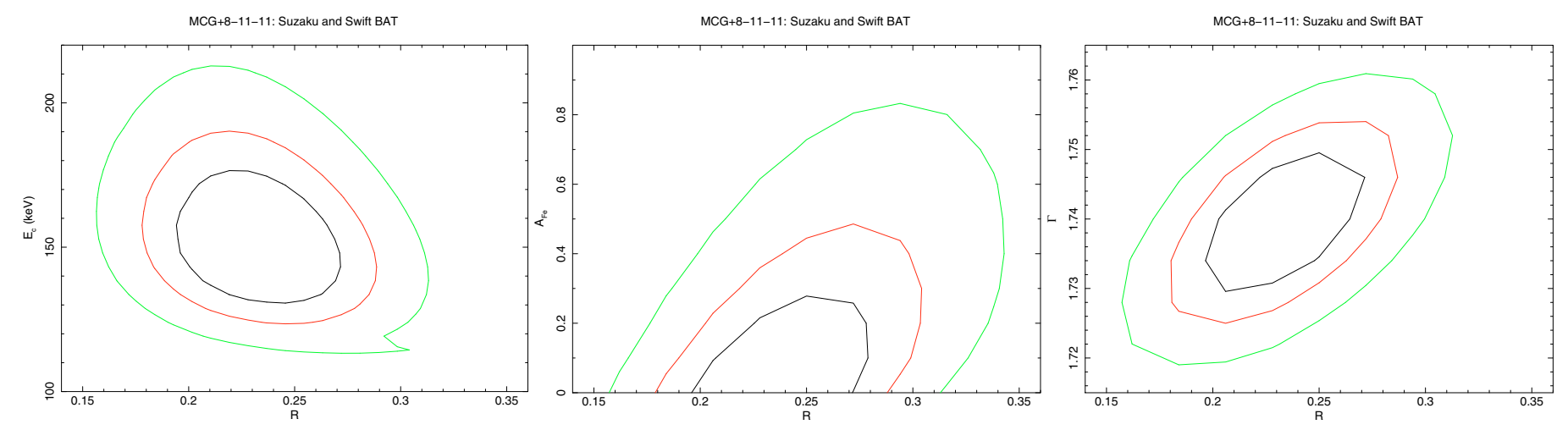

Fig. 6. Suzaku+Swift BAT best fit contour plots (black, red, green lines correspond to 68, 90, and 99\% confidence level for two interesting parameters). Left: Compton reflection amount $R$ vs. cutoff energy $E_{\mathrm{c}}$; middle: Compton reflection amount $R$ vs. iron abundance $A_{\mathrm{Fe}} ;$ right: Compton reflection amount $R$ vs. spectral photon index $\Gamma$.

is negligible $(R<0.1)$. Therefore, all the narrow component of the iron $\mathrm{K} \alpha$ line is now modelled by emission from the BLR ( $\sigma=30 \mathrm{eV}$ as above), with an $E W \simeq 70 \mathrm{eV}$. The inner radius of the accretion disc is just beyond the innermost stable orbit for a non-spinning $\mathrm{BH}\left(13_{-4}^{+31} r_{\mathrm{g}}\right)$.

The resulting scenario is very interesting. If the reflection component originates entirely in the accretion disc, consistently with the observed relativistic component of the iron $\mathrm{K} \alpha$ line, it is unavoidable that the narrow core of the emission line is produced in Compton-thin material, such as the BLR, whose contribution to the reflection amount would be negligible. This, in turns, implies that there would be no evidence of the classical Comptonthick torus in this source. Given the high-flux state of the source during the Suzaku observation (see Sect. 3.3 and Fig. 5), it is possible that the reprocessing components from the torus are diluted by the stronger primary continuum. Although this would justify the low (or absent) Compton reflection from the torus, most of the narrow iron $\mathrm{K} \alpha$ line would still be produced in Comptonthin material, because the component originating from the torus would be diluted.

This situation is similar to the one observed in another Seyfert galaxy, NGC 7213, the only Seyfert 1 galaxy with a negligible amount of Compton reflection (Bianchi et al. 2003, 2008b; Lobban et al. 2010). The observed iron neutral line in this source $(E W \simeq 100 \mathrm{eV})$ was explained by Bianchi et al. (2008b) as being produced mainly in the BLR, as confirmed by the consistency between the FWHM of the broad component of the $\mathrm{H} \alpha$ line and the neutral iron $\mathrm{K} \alpha$ line, in quasi-simultaneous optical/Chandra observations. The same scenario would apply to MCG+8-11-11, but with the notable difference that this object exhibits spectral signatures of an accretion disc.

We found two $\mathrm{BH}$ mass estimates for $\mathrm{MCG}+8-11-11$ in the literature, both derived by means of the BLR radius vs. $5100 \AA$ relationship, and the $\mathrm{H} \beta$, but using different data, i.e. $1.5 \times 10^{7} M_{\odot}\left(\right.$ Bian \& Zhao 2003) and $1.2 \times 10^{8} M_{\odot}$ (Winter et al. 2010). Applying a bolometric correction of 30 to the observed $2-$ $10 \mathrm{keV}$ luminosity (as appropriate in this luminosity range, according to Marconi et al. 2004), we recover $L_{\text {bol }} / L_{\text {edd }} \simeq 0.1-1$. Although a better $\mathrm{BH}$ mass estimate is needed to assess the reliability of this accretion rate, the difference with respect to NGC 7213 is clear, being it characterised by a low accretion rate $\left(\simeq 3 \times 10^{-3}\right.$ : Bianchi et al. 2009a). This could explain the lack of relativistic signatures in the latter, where the accretion disc may be truncated and its inner part replaced by some sort of inefficient disc (see e.g. Lobban et al. 2010). The absence of the torus in both sources is instead more puzzling. The formation of the torus may be suppressed at low accretion rates and/or luminosities, as predicted for the BLR (e.g. Nicastro 2000; Elitzur $\&$ Shlosman 2006). However, as we have already seen, the accretion rates of these two sources have nothing in common, and their luminosities are by no means low.

\section{Conclusions}

We have presented a long Suzaku observation of one of the X-ray brightest AGN, MCG+8-11-11. The spectrum is characterised by a standard power-law photon index of 1.7-1.8, and a warm absorber which cannot be satisfactorily modelled with a single gas component. The notable absence of a soft excess, which is nearly ubiquitous in unobscured Seyfert galaxies, may be partly due to the high Galactic column density along the line-of sight.

The fits performed in the $0.6-10 \mathrm{keV}$ band provide results consistent with a previous XMM-Newton observation, i.e. a large Compton reflection component $(R \simeq 1)$ and the absence of a relativistic component of the neutral iron $\mathrm{K} \alpha$ emission line. However, when the PIN data are included, the reflection amount drops significantly $(R \simeq 0.2-0.3)$, and a relativistic iron line is required, the latter confirmed by a phenomenological analysis in a restricted energy band (3-10 keV). The addition of the Swift BAT 54-month spectrum up to $150 \mathrm{keV}$ allowed us to measure an high-energy cutoff at $150 \mathrm{keV}$, in agreement with previous results.

When a self-consistent model is applied to the whole data, the observed reflection component appears to be associated entirely with the relativistic component of the iron $\mathrm{K} \alpha$ line. Therefore, all reprocessing from Compton-thick material must be associated with the accretion disc, and no evidence for the classical pc-scale torus is found. It is unavoidable that the narrow core of the neutral iron $\mathrm{K} \alpha$ line is produced in Compton-thin material, such as the BLR, whose contribution to the reflection amount would be negligible. Although more model-dependent, this situation is similar to the one observed in another Seyfert galaxy, NGC 7213, the only Seyfert 1 galaxy with a negligible amount of Compton reflection, but with the notable difference that MCG+8-11-11 exhibits spectral signatures of an accretion disc. The very low accretion rate of NGC 7213 could explain the lack of relativistic signatures in its spectrum, but the absence of the torus in both sources is more difficult to explain, since their luminosities are comparable, and their accretion rates are completely different. 
Acknowledgements. This research has made use of data obtained from the Suzaku satellite, a collaborative mission between the space agencies of Japan (JAXA) and the USA (NASA). We thank the anonymous referee for useful suggestions which improved the clarity of the paper. S.B., E.P. and G.M. acknowledge financial support from ASI (grant I/088/06/0). We would like to thank K. Nandra, G. Miniutti, K. Arnaud and C. Gordon for the XSPEC model PEXMON, and K. Hamaguchi for his support on Suzaku data reduction and analysis.

\section{References}

Anders, E., \& Grevesse, N. 1989, Geochim. Cosmochim. Acta, 53, 197 Antonucci, R. 1993, ARA\&A, 31, 473

Arnaud, K. A. 1996, in Astronomical Data Analysis Software and Systems V, ASP Conf. Ser., 101, 17

Balucinska-Church, M., \& McCammon, D. 1992, ApJ, 400, 699

Bearden, J. A. 1967, Rev. Mod. Phys., 39, 78

Beckmann, V., Soldi, S., Ricci, C., et al. 2009, A\&A, 505, 417

Bian, W., \& Zhao, Y. 2003, MNRAS, 343, 164

Bianchi, S., Matt, G., Balestra, I., \& Perola, G. C. 2003, A\&A, 407, L21

Bianchi, S., Matt, G., Nicastro, F., Porquet, D., \& Dubau, J. 2005, MNRAS, 357, 599

Bianchi, S., Guainazzi, M., Matt, G., \& Fonseca Bonilla, N. 2007, A\&A, 467, L19

Bianchi, S., Corral, A., Panessa, F., et al. 2008a, MNRAS, 385, 195

Bianchi, S., La Franca, F., Matt, G., et al. 2008b, MNRAS, 389, L52

Bianchi, S., Guainazzi, M., Matt, G., Fonseca Bonilla, N., \& Ponti, G. 2009a, A\&A, 495, 421

Bianchi, S., Piconcelli, E., Chiaberge, M., et al. 2009b, ApJ, 695, 781

de la Calle Pérez, I., Longinotti, A. L., Guainazzi, M., et al. 2010, A\&A, in press
Dickey, J. M., \& Lockman, F. J. 1990, ARA\&A, 28, 215

Dovčiak, M., Karas, V., \& Yaqoob, T. 2004, ApJS, 153, 205

Elitzur, M., \& Shlosman, I. 2006, ApJ, 648, L101

Elvis, M., Risaliti, G., Nicastro, F., et al. 2004, ApJ, 615, L25

Fabian, A. C., Iwasawa, K., Reynolds, C. S., \& Young, A. J. 2000, PASP, 112, 1145

Ferland, G. J., Korista, K. T., Verner, D. A., et al. 1998, PASP, 110, 761

Grandi, P., Haardt, F., Ghisellini, G., et al. 1998, ApJ, 498, 220

Kalberla, P. M. W., Burton, W. B., Hartmann, D., et al. 2005, A\&A, 440, 775

Lobban, A. P., Reeves, J. N., Porquet, D., et al. 2010, MNRAS, 408, 551

Longinotti, A. L., de La Calle, I., Bianchi, S., Guainazzi, M., \& Dovčiak, M. 2008, Mem. Soc. Astron. Ital., 79, 259

Marconi, A., Risaliti, G., Gilli, R., et al. 2004, MNRAS, 351, 169

Matt, G. 2002, MNRAS, 337, 147

Matt, G., Bianchi, S., de Rosa, A., Grandi, P., \& Perola, G. C. 2006, A\&A, 445, 451

Nandra, K., O’Neill, P. M., George, I. M., \& Reeves, J. N. 2007, MNRAS, 382, 194

Nicastro, F. 2000, ApJ, 530, L65

Nicastro, F., Fiore, F., Perola, G. C., \& Elvis, M. 1999, ApJ, 512, 184

Osterbrock, D. E., \& Ferland, G. J. 2006, Astrophysics of gaseous nebulae and active galactic nuclei, ed. G. J. Osterbrock, \& D. E. Ferland

Osterbrock, D. E., \& Shuder, J. M. 1982, ApJS, 49, 149

Panessa, F., Carrera, F. J., Bianchi, S., et al. 2009, MNRAS, 398, 1951

Perola, G. C., Matt, G., Fiore, F., et al. 2000, A\&A, 358, 117

Perola, G. C., Matt, G., Cappi, M., et al. 2002, A\&A, 389, 802

Piconcelli, E., Jimenez-Bailón, E., Guainazzi, M., et al. 2005, A\&A, 432, 15

Puccetti, S., Fiore, F., Risaliti, G., et al. 2007, MNRAS, 377, 607

Risaliti, G., Elvis, M., Fabbiano, G., Baldi, A., \& Zezas, A. 2005, ApJ, 623, L93

Winter, L. M., Lewis, K. T., Koss, M., et al. 2010, ApJ, 710, 503 